Pacific Journal of Mathematics

TENSOR PRODUCTS OF COMPACT CONVEX SETS 


\title{
TENSOR PRODUCTS OF COMPACT CONVEX SETS
}

\author{
ISAAC NAMIOKA AND R. R. Phelps
}

\begin{abstract}
Suppose that $K_{1}$ and $K_{2}$ are compact convex subsets of locally convex spaces $E_{1}$ and $E_{2}$ respectively. There are several definitions of new compact convex sets associated with $K_{1}$ and $K_{2}$, each of which may reasonably be called a "tensor product" of $K_{1}$ and $K_{2}$. We compare these different tensor products and their extreme points; in doing so, we obtain some new characterizations of Choquet simpiexes, another formulation of Grothendieck's approximation problem and much simpler proofs of known characterizations of the extreme points of these tensor products. Most of these results are obtained as special cases of theorems in the first half of the paper which deal with the state spaces of tensor products of partially ordered linear spaces with order unit.
\end{abstract}

1. Tensor products of partially ordered spaces. A partially ordered, linear space with order unit is a triple $(E, P, u)$, where the linear space $E$ is given the partial ordering induced by the cone $P$, where $P \cap(-P)=\{0\}$, and where $u$ is an order unit for $P$, i.e., $P-u$ absorbs $E$. Given a partially ordered linear space $(E, P)$, the dual cone $P^{\star}$ is the space of all linear functionals on $E$ which are nonnegative on $P$. The subspace of the algebraic dual of $E$ which is generated by $P^{\star}$ is denoted by $E^{\star}$; it is clear that $E^{\star}=P^{\star}-P^{\star}$. The partially ordered linear space $\left(E^{\star}, P^{\star}\right)$ is called the order dual of $(E, P)$.

If $\left(E_{1}, P_{1}, u_{1}\right)$ and $\left(E_{2}, P_{2}, u_{2}\right)$ are two partially ordered linear spaces with order units, then in the tensor product $E_{1} \otimes E_{2}$ the cone generated by elements of the form $x_{1} \otimes x_{2}\left(x_{i} \in P_{i}\right)$ will be denoted by $P_{1} \otimes P_{2}$. The triple $\left(E_{1} \otimes E_{2}, P_{1} \otimes P_{2}, u_{1} \otimes u_{2}\right)$ is a partially ordered linear space with order unit $[3,8]$.

Given a partially ordered linear space with order unit $(E, P, u)$, its state space $S$ is the set of all $f$ in $P^{\star}$ such that $\langle f, u\rangle=1$, provided with the weak ${ }^{\star}\left(=w\left(E^{\star}, E\right)\right)$ topology. Clearly, $S$ is convex compact and Hausdorff. It is possible for $S$ to be empty (cf. [7, p. 26]). If $S$ is the state space of $\left(E_{1} \otimes E_{2}, P_{1} \otimes P_{2}, u_{1} \otimes u_{2}\right)$ and $s \in S$, then there exists a related functional $s_{1}$ on $E_{1}$ defined by

$$
\left\langle s_{1}, x_{1}\right\rangle=\left\langle s, x_{1} \otimes u_{2}\right\rangle, \quad x_{1} \in E_{1} \text {. }
$$

It is clear that $s_{1}$ is in the state space $S_{1}$ of $\left(E_{1}, P_{1}, u_{1}\right)$, and it is clear how to define the analogous state $s_{2}$ in $S_{2}$. In the reverse direction, suppose that $t_{i}$ is in the state space $S_{i}$ of $\left(E_{i}, P_{i}, u u_{i}\right)$ and define the functional $t_{1} \otimes t_{2}$ on $E_{1} \otimes E_{2}$ by setting 


$$
\left\langle t_{1} \otimes t_{2}, x_{1} \otimes x_{2}\right\rangle=\left\langle t_{1}, x_{1}\right\rangle\left\langle t_{2}, x_{2}\right\rangle, \quad x_{i} \in E_{i},
$$

and extending linearly. The functional $t_{1} \otimes t_{2}$ is clearly in the state space $S$ of $\left(E_{1} \otimes E_{2}, P_{1} \otimes P_{2}, u_{1} \otimes u_{2}\right)$.

It is known $[1,3,4]$ that if $S_{1}$ and $S_{2}$ are simplexes (see below), then a state $s$ of $S$ is an extreme point of $S$ if and only if $s=s_{1} \otimes s_{2}$, and each $s_{i}$ is extreme in $S_{i}$. Our first few results show the extent to which this remains true without making any assumptions about the sets $S_{1}$ and $S_{2}$.

Lemma 1.1. Let $S_{1}, S_{2}$ and $S$ be the state spaces of the partially ordered linear spaces with order unit $\left(E_{1}, P_{1}, u_{1}\right),\left(E_{2}, P_{2}, u_{2}\right)$ and $\left(E_{1} \otimes E_{2}, P_{1} \otimes P_{2}, u_{1} \otimes u_{2}\right)$ respectively. If $s \in S$ and if $s_{1}$ is an extreme point of $S_{1}$, then $s=s_{1} \otimes s_{2}$.

Proof. Fix an element $x_{2}$ of $E_{2}$, with $0 \leqq x_{2} \leqq u_{2}$, and define the functional $f$ on $E_{1}$ by $\left\langle f, x_{1}\right\rangle=\left\langle s, x_{1} \otimes x_{2}\right\rangle$. Since $0 \leqq f \leqq s_{1}$ and since $s_{1}$ is extreme in $S_{1}$, there exists a constant (necessarily equal to $\left\langle f, u_{1}\right\rangle$ ) such that $f=\left\langle f, u_{1}\right\rangle s_{1}$. Thus, for any $x_{1}$ in $E_{1}$, we have $\left\langle s, x_{1} \otimes x_{2}\right\rangle=\left\langle f, x_{1}\right\rangle=\left\langle f, u_{1}\right\rangle\left\langle s_{1}, x_{1}\right\rangle=\left\langle s_{2}, x_{2}\right\rangle\left\langle s_{1}, x_{1}\right\rangle$. It follows easily from this that $s=s_{1} \otimes s_{2}$.

If $K$ is a nonempty compact convex subset of a locally convex space $E$, let $M_{1}(K)$ denote the space of all regular Borel probability measures on $K$ and let $Q(K)$ denote the subspace of all maximal such measures on $K$ [9]. We denote by $r$ the affine resultant mapping from $M_{1}^{-}(K)$ onto $K$; it is characterized by the equation

$$
f(r(\mu))=\int_{K} f d \mu, \quad f \in E, \mu \in M_{1}^{+}(K) .
$$

The restriction $r_{m}$ of $r$ to the maximal measures $Q(K)$ is also onto, and $K$ is called a simplex if $r_{m}$ is one-to-one. We will say that $K$ is simplex-like if there exists an affine map $\sigma: K \rightarrow M_{1}^{+}(K)$ such that $r \cdot \sigma$ is the identity map on $K$. (Note that we are not assuming any continuity properties for the affine cross-section $\sigma$.) If $K$ is a simplex, then the inverse of $r_{m}$ is such an affine cross-section, so every simplex is simplex-like. Eventually (Theorem 1.4), we will prove the converse to this last assertion.

We denote the set of extreme points of a convex set $K$ by $\partial K$.

THEOREM 1.2. Let $S_{1}, S_{2}$ and $S$ be the state spaces of $\left(E_{1}, P_{1}, u_{1}\right)$, $\left(E_{2}, P_{2}, u_{2}\right)$ and $\left(E_{1} \otimes E_{2}, P_{1} \otimes P_{2}, u_{1} \otimes u_{2}\right)$ respectively. If $S_{1}$ is simplex-like, then for each $s$ in $\partial S$ we have $s=s_{1} \otimes s_{2}$ and $s_{i} \in \hat{\partial} S_{i}$. 
Proof. Let $\sigma$ be an affine cross-section for $r$. These maps can be extended to linear maps $\bar{r}: M\left(S_{1}\right) \rightarrow E_{1}^{\star}$ and $\bar{\sigma}: E_{1}^{\star} \rightarrow M\left(S_{1}\right)$ so that $\bar{r} \cdot \bar{\sigma}$ is the identity on $E_{1}^{\star}, \bar{r} \geqq 0$ and $\bar{\sigma} \geqq 0$, where $M\left(S_{1}\right)$ is the space of all signed regular Borel measures on $S_{1}$. Given any $t$ in $S$, we can define a linear map $T_{t}: E_{2} \rightarrow E_{1}^{\star}$ by $\left\langle T_{t}\left(x_{2}\right), x_{1}\right\rangle=\left\langle t, x_{1} \otimes x_{2}\right\rangle$. This map satisfies

$$
\text { (a) } T_{t} \geqq 0 \text { and (b) } T_{t}\left(u_{2}\right)\left(=t_{1}\right) \in S_{1} \text {. }
$$

Conversely, given any linear $T: E_{2} \rightarrow E_{1}^{\star}$ which satisfies (a) and (b), there exists a unique $t$ in $S$ such that $T=T_{t}$. The correspondence $t \leftrightarrow T_{t}$ is an affine isomorphism, so if $s \in \partial S$, then $T_{s}$ is extreme in the set of operators from $E_{2}$ to $E_{1}^{\star}$ which satisfy (a) and (b). Given $s$ in $\partial S$, then, let $\mu=\sigma\left(s_{1}\right) \in M_{1}^{+}\left(S_{1}\right)$, i. e., $\mu=\sigma T_{s}\left(u_{2}\right)$. Let $j$ be the injection map $L^{\infty}\left(S_{1}, \mu\right) \rightarrow M\left(S_{1}\right)$ defined by $j(f)=f \mu\left(f \in L^{\infty}\left(S_{1}, \mu\right)\right)$. Since $u_{2}$ is an order unit, given $x_{2}$ in $E_{2}$ there is a positive number $m$ such that $-m u_{2} \leqq x_{2} \leqq m u_{2}$ and hence $-m \mu \leqq \bar{\sigma} T_{s}\left(x_{2}\right) \leqq m \mu$. By the Radon-Nikodym theorem there is a unique element $L\left(x_{2}\right)$ of $L^{\infty}\left(S_{1}, \mu\right)$ such that $L\left(x_{2}\right) \mu=j L\left(x_{2}\right)=\bar{\sigma} T_{s}\left(x_{2}\right)$. The map $L: E_{2} \rightarrow L^{\infty}\left(S_{1}, \mu\right)$ is clearly linear, positive and $L\left(u_{2}\right)=1$.

We wish to show that $s_{1} \in \partial S_{1}$. If not, then $s_{1}=1 / 2\left(t_{1}+t_{1}^{\prime}\right)$, where $t_{1} \neq t_{1}^{\prime}$ and $t_{1}, t_{1}^{\prime} \in S_{1}$. Define linear maps $T, T^{\prime}$ from $E_{2}$ into $E_{1}^{\star}$ by $T x_{2}=\bar{r}\left[L\left(x_{2}\right) \sigma\left(t_{1}\right)\right], T^{\prime} x_{2}=\bar{r}\left[L\left(x_{2}\right) \sigma\left(t_{1}^{\prime}\right)\right]$. (Since $0 \leqq \sigma\left(t_{1}\right) \leqq 2 \mu$, for instance, $L\left(x_{2}\right) \sigma\left(t_{1}\right)$ is well-defined.) Clearly, $T, T^{\prime} \geqq 0$ and $T\left(u_{2}\right)=t_{1}$, $T^{\prime}\left(u_{2}\right)=t_{1}^{\prime}$. Thus, $T$ and $T^{\prime}$ satisfy (a) and (b), and $T\left(u_{2}\right) \neq T^{\prime}\left(u_{2}\right)$, so $T \neq T^{\prime}$. Moreover, for every $x_{2}$ in $E_{2}, 1 / 2\left(T+T^{\prime}\right)\left(x_{2}\right)=\bar{r}\left[L\left(x_{2}\right) \sigma\left(s_{1}\right)\right]=$ $\bar{r}\left[L\left(x_{2}\right) \mu\right]=\bar{r} \bar{\sigma}\left[T_{s}\left(x_{2}\right)\right]=T_{s}\left(x_{2}\right)$, contradicting the fact that $T_{s}$ is extreme. We now know that $s_{1} \in \partial S_{1}$, and Lemma 1.1 shows that $s=s_{1} \otimes s_{2}$. It is straight-forward to check that $s_{2} \in \partial S_{2}$, so the proof is complete.

The following proposition was proved in [1, 3, 4] under the additional hypothesis that each $S_{1}$ was a simplex.

Proposition 1.3. Let $S_{1}, S_{2}$ and $S$ denote state spaces as in Lemma 1.1. If $s_{i} \in \partial S_{i}(i=1,2)$, then $s_{1} \otimes s_{2} \in \partial S$.

Proof. Suppose that $t$ is a linear functional on $E_{1} \otimes E_{2}$, that $t_{1}$ and $t_{2}$ are defined in the obvious way, and that $s_{1} \otimes s_{2} \pm t \in S$; we want to show that $t=0$. Since $s_{1} \in \partial S_{1}$ and since $s_{1} \pm t_{1} \in S_{1}$, we see that $t_{1}=0$. Suppose that $x_{1} \in E_{1}$, with $0 \leqq x_{1} \leqq u_{1}$. If $x_{2} \in P_{2}$, then $0 \leqq x_{1} \otimes x_{2} \leqq u_{1} \otimes x_{2}$ and hence

$$
\begin{aligned}
0 & \leqq\left\langle s_{1} \otimes s_{2}, x_{1} \otimes x_{2}\right\rangle \pm\left\langle t, x_{1} \otimes x_{2}\right\rangle \\
& \leqq\left\langle s_{1} \otimes s_{2}, u_{1} \otimes x_{2}\right\rangle \pm\left\langle t, x_{1} \otimes x_{2}\right\rangle=\left\langle s_{2}, x_{2}\right\rangle \pm\left\langle t, x_{1} \otimes x_{2}\right\rangle .
\end{aligned}
$$


Since $s_{2}$ is extreme (and since $\left\langle t, x_{1} \otimes u_{2}\right\rangle=0$ ), this shows that $\left\langle t, x_{1} \otimes x_{2}\right\rangle=0$ for all $x_{2}$ in $E_{2}$. Finally, since $E_{1} \otimes E_{2}$ is generated by elements of the form $x_{1} \otimes x_{2}$, where $x_{i} \in P_{\imath}$, we conclude that $t=0$.

[The special case of [3; Th. 3.1], where all the $\Gamma_{a}$ are trivial, follows easily from Theorem 1.2 and Proposition 1.3. By a very small modification of Theorem 1.2, one can prove Theorem 3.1 of [3] in its full generality. We leave such a modification of Theorem 1.2 (and its proof) to the reader.]

Given two partially ordered linear spaces with order unit $\left(E_{1}, P_{1}, u_{1}\right)$ and $\left(E_{2}, P_{2}, u_{2}\right)$, there is a second ordering one can introduce on $E_{1} \otimes E_{2}$ (cf. [3, 8]) which may be described as follows: Note first that the spaces $E_{1} \otimes E_{2}$ and $E_{1}^{\star} \otimes E_{2}^{\star}$ are paired by (the linear extension of) the obvious rule $\left\langle f_{1} \otimes f_{2}, x_{1} \otimes x_{2}\right\rangle=\left\langle f_{1}, x_{1}\right\rangle\left\langle f_{2}, x_{2}\right\rangle$. We define a second cone $P^{\wedge}$ by

$$
P^{\wedge}=\left\{x \in E_{1} \otimes E_{2}:\langle f, x\rangle \geqq 0 \text { for all } f \text { in } P_{1}^{\star} \otimes P_{2}^{\star}\right\} .
$$

It is clear that $P_{1} \otimes P_{2} \subset P^{\wedge}$ and that $u_{1} \otimes u_{2}$ is an order unit for $P^{\wedge}$ (since it is one for $P_{1} \otimes P_{2}$ ). Let $S$ and $S^{\wedge}$ be the state spaces of $\left(E_{1} \otimes E_{2}, P_{1} \otimes P_{2}, u_{1} \otimes u_{2}\right)$ and $\left(E_{1} \otimes E_{2}, P^{\wedge}, u_{1} \otimes u_{2}\right)$ respectively. It is evident that $S^{\wedge} \subset S$, and the following theorem gives conditions under which $S^{\wedge}=S$. The validity of “(c) implies (a)" was suggested to us by E. Effros.

Theorem 1.4. Let $S_{1}$ be the state space of $\left(E_{1}, P_{1}, u_{1}\right)$; then the following assertions are equivalent

(a) $S_{1}$ is a simplex.

(b) $S_{1}$ is simplex-like.

(c) For any partially ordered linear space with order unit $\left(E_{2}, P_{2}, u_{2}\right)$, the two state spaces resulting from the two orderings on $E_{1} \otimes E_{2}$ coincide.

Proof. We have already noted that (a) implies (b). Denoting the two state spaces by $S$ and $S^{\wedge}$ as above, Theorem 1.2 shows that if $S_{1}$ is simplex-like, then every extreme point of $S$ is of the form $s_{1} \otimes s_{2}$. Since such functionals are clearly in $S^{\wedge}$, we have $\partial S \subset S^{\wedge} \subset S$. By the Krein-Milman theorem, then, $S=S^{\wedge}$. It remains to prove that (c) implies (a). Let $E=R^{3}$, let $e_{1}, e_{2}, e_{3}$ be the usual unit basis vectors and let $P$ be the cone in $E$ generated by the vectors $e_{1}, e_{2}, e_{3}$ and $e_{1}+e_{2}-e_{3}$. For an order unit $u$ we may choose any point in the interior of $P$ (e.g., $\left.u=e_{1}+e_{2}\right)$. The order dual $\left(E^{\star}, P^{\star}\right)$ is again isomorphic to $R^{3}$, and if $d_{1}, d_{2}, d_{3}$ are basis vectors dual to $e_{1}, e_{2}, e_{3}$, then $P^{\star}$ is generated by $d_{1}, d_{2}, d_{1}+d_{3}, d_{2}+d_{3}$. (Equivalently, $P$ is the intersection of the half-spaces through the origin defined by these four 
functionals.) Let $S$ and $S^{\wedge}$ be the state spaces of $\left(E_{1} \otimes E, P_{1} \otimes P\right.$, $\left.u_{1} \otimes u\right)$ and $\left(E_{1} \otimes E, P^{\wedge}, u_{1} \otimes u\right)$ respectively, and suppose that $S=S^{\wedge}$. We will show that $\left(E_{1}^{\star}, P_{1}^{\star}\right)$ is a vector lattice, which is equivalent to showing that $S_{1}$ is a simplex (c.f. [9].).

It is clear that the algebraic dual of $E_{1} \otimes E$ can be identified with $E_{1}^{\prime} \otimes E^{\star}$ (where $E_{1}^{\prime}$ is the algebraic dual of $E_{1}$ ) by means of the usual pairing between $E_{1} \otimes E$ and $E_{1}^{\prime} \otimes E^{\star}$, and each element of $E_{1}^{\prime} \otimes E^{\star}$ can be represented uniquely as $f_{1} \otimes d_{1}+f_{2} \otimes d_{2}+f_{3} \otimes d_{3}$, where $f_{i} \in E_{1}^{\prime}$. Such an element is in $\left(P_{1} \otimes P\right)^{\star}$ if and only if $f_{i} \geqq 0$ $(i=1,2,3)$ and $f_{1}+f_{2}-f_{3} \geqq 0$. It is immediate from the definition of $P^{\wedge}$ and the separation theorem that $\left(P^{\wedge}\right)^{\star}$ is the $w\left(E_{1}^{\prime} \otimes E^{\star}, E_{1} \otimes E\right)$ closure of $P_{1}^{\star} \otimes P^{\star}$ in $E_{1}^{\prime} \otimes E^{\star}$. We claim that $P_{1}^{\star} \otimes P^{\star}$ is, in fact, $w\left(E_{1}^{\prime} \otimes E^{\star}, E_{1} \otimes E\right)$-closed. Indeed, an arbitrary element of $P_{1}^{\star} \otimes P^{\star}$ may be written (not uniquely) in the form $g_{1} \otimes d_{1}+g_{2} \otimes d_{2}+g_{3} \otimes$ $\left(d_{1}+d_{3}\right)+g_{4} \otimes\left(d_{2}+d_{3}\right)$, where $g_{i} \in P_{1} \quad(i=1,2,3,4)$. Suppose that there is a net $\left\{g^{\alpha}\right\}=\left\{g_{1}^{\alpha} \otimes d_{1}+g_{2}^{\alpha} \otimes d_{2}+g_{3}^{\alpha} \otimes\left(d_{1}+d_{3}\right)+g_{4}^{\alpha} \otimes\left(d_{2}+d_{3}\right)\right\}$ of such elements, converging to $f_{1} \otimes d_{1}+f_{2} \otimes d_{2}+f_{3} \otimes d_{3}$ in the $w\left(E_{1}^{\prime} \otimes E^{\star}, E_{1} \otimes E\right)$ topology. Then $g_{1}^{\alpha}+g_{3}^{\alpha}$ converges to $f_{1}$ in the weak ${ }^{\star}$ topology in $E_{1}^{\star}$, hence (in particular) $\left\langle g_{1}^{\alpha}, u_{1}\right\rangle+\left\langle g_{3}^{\alpha}, u_{1}\right\rangle \rightarrow\left\langle f_{1}, u_{1}\right\rangle$. Since $0 \leqq\left\langle g_{\imath}^{\alpha}, u_{1}\right\rangle$, the net $\left\{\left\langle g_{1}^{\alpha}, u_{1}\right\rangle\right\}$ is eventually bounded. But the set $\left\{f: f \in P_{1},\left\langle f, u_{1}\right\rangle \leqq M\right\}$ is weak ${ }^{\star}$ compact, and hence $\left\{g_{1}^{\alpha}\right\}$ has a convergent subnet. Without loss of generality we may assume that $\lim g_{1}^{\alpha}=g_{1}$ exists. It then follows easily that $\lim g_{i}^{\alpha}=g_{i}$ exist for $i=1,2,3,4$, and since $P_{1}^{\star}$ is weak ${ }^{\star}$ closed, $g_{i} \in P_{1}^{\star}(i=1,2,3,4)$. Thus, $\lim g^{\alpha}=g_{1} \otimes d_{1}+g_{2} \otimes d_{2}+g_{3} \otimes\left(d_{1}+d_{3}\right)+g_{4} \otimes\left(d_{2}+d_{3}\right)$ is in $P_{1}^{\star} \otimes P^{\star}$, which shows that the latter is $w\left(E_{1}^{\prime} \otimes E^{\star}, E_{1} \otimes E\right)$-closed. Consequently, $\left(P^{\wedge}\right)^{\star}=P_{1}^{\star} \otimes P^{\star}$.

Now, since $S=S^{\wedge}$, we have $\left(P_{1} \otimes P\right)^{\star}=\left(P^{\wedge}\right)^{\star}=P_{1}^{\star} \otimes P^{\star}$. Thus, given $f_{1}, f_{2}, f_{3}$ in $P_{1}^{\star}$ such that $f_{3} \leqq f_{1}+f_{2}$, there exist elements $g_{1}, g_{2}$, $g_{3}, g_{4}$ in $P_{1}^{\star}$ such that

$$
\begin{aligned}
f_{1} \otimes d_{1}+f_{2} \otimes d_{2}+f_{3} \otimes d_{3} & =g_{1} \otimes d_{1}+g_{2} \otimes d_{2}+g_{3} \otimes\left(d_{1}+d_{3}\right)+g_{4} \otimes\left(d_{2}+d_{3}\right) \\
& =\left(g_{1}+g_{3}\right) \otimes d_{1}+\left(g_{2}+g_{4}\right) \otimes d_{2}+\left(g_{3}+g_{4}\right) \otimes d_{3} .
\end{aligned}
$$

This shows that $f_{1}=g_{1}+g_{3}, f_{2}=g_{2}+g_{4}$ and $f_{3}=g_{3}+g_{4}$, i.e., the space $\left(E_{1}^{\star}, P_{1}^{\star}\right)$ has the Riesz decomposition property (cf. [7, p. 27]). The following lemma then completes the proof.

LEMma 1.5. Let $(E, P)$ be a partially ordered linear space such that $E=P-P$. Then $\left(E^{\star}, P^{\star}\right)$ is a vector lattice if and only if it has the Riesz decomposition property.

Proof. Every vector lattice has the decomposition property, so we want to prove the converse. By a standard argument, the decom- 
position property implies that if $g_{1}, g_{2} \leqq f_{1}, f_{2}$ in $E^{\star}$, then there exists $h$ in $E^{\star}$ such that $g_{1}, g_{2} \leqq h \leqq f_{1}, f_{2}$. Thus, if $f_{1}, f_{2} \in P^{\star}$, then the set $A=\left\{g: 0 \leqq g \leqq f_{1}, f_{2}\right\}$ is directed by $\leqq$. For each $x$ in $P$, the net $\{\langle g, x\rangle: g \in A\}$ is monotone and bounded, hence $\lim \{\langle g, x\rangle: g \in A\}$ exists. Since $P-P=E$, the net $\{g: g \in A\}$ converges pointwise to a positive functional on $E$, which is readily verified to be $f_{1} \wedge f_{2}$. This suffices (cf. e.g., [9, p. 60]) to show that $E^{\star}$ is a lattice.

2. Tensor products of compact convex sets. Suppose that $K$ is a compact convex set (always assumed to be a nonempty subset of some locally convex Hausdorff space) and let $A(K)$ (or simply $A$ ) denote the space of all real-valued continuous affine functions on $K$. If $A^{+}$denotes the cone of nonnegative functions in $A$ and 1 denotes the function identically equal to 1 , then $\left(A, A^{+}, 1\right)$ is a partially ordered linear space with order unit. Now $A$ is a Banach space under the supremum norm and the order dual of $\left(A, A^{+}\right)$is precisely the space $A^{*}$ of all continuous linear functionals on $A$, ordered in the usual way (cf. [7, p. 45]). Under the evaluation mapping, $K$ is affinely homeomorphic to the state space of $\left(A, A^{+}, 1\right)$.

Suppose that $K_{1}$ and $K_{2}$ are compact convex subsets of locally convex Hausdorff spaces, let $A_{i}=A\left(K_{i}\right)$ and consider the partial orderings on $A_{1} \otimes A_{2}$ induced by the cones $A_{1}^{+} \otimes A_{2}^{+}$and $P^{\wedge}$ defined in $\S 1$. Let $K_{1} \square K_{2}$ denote the state space $S$ of $\left(A_{1} \otimes A_{2}, A_{1}^{+} \otimes A_{2}^{+}, 1 \otimes 1\right)$ and let $K_{1} \triangle K_{2}$ denote the state space $S^{\wedge}$ of $\left(A_{1} \otimes A_{2}, P^{\wedge}, 1 \otimes 1\right)$. As noted before, the inclusion $K_{1} \triangle K_{2} \subset K_{1} \square K_{2}$ is always valid.

If $x_{i} \in K_{i}$, then (as before) $x_{1} \otimes x_{2}$ is defined to be the element in $K_{1} \triangle K_{2} \subset K_{1} \square K_{2}$ which satisfies $\left\langle x_{1} \otimes x_{2}, f_{1} \otimes f_{2}\right\rangle=f_{1}\left(x_{1}\right) f_{2}\left(x_{2}\right),\left(f_{i} \in A_{i}\right)$. The following theorem is a consequence of Theorem 1.2 and Proposition 1.3. A complete description of the extreme points of $K_{1} \square K_{2}$ remains an open problem.

Theorem 2.1. If $x_{i} \in \partial K_{i}(i=1,2)$, then $x_{1} \otimes x_{2}$ is extreme in $K_{1} \square K_{2}$. If $K_{1}$ is a simplex, then any $x$ in $\partial\left(K_{1} \square K_{2}\right)$ is of the form $x=x_{1} \otimes x_{2}$, where $x_{i} \in \partial K_{i}$.

Theorem 1.4 yields the following result. (The implication " $(d)$ implies (a)" is contained in the proof of Theorem 1.4)

THEOREM 2.2. The following assertions about a compact convex set $K_{1}$ are equivalent:

(a) $K_{1}$ is a simplex.

(b) $K_{1}$ is simplex-like.

(c) $K_{1} \square K_{2}=K_{1} \triangle K_{2}$ for every compact convex set $K_{2}$.

(d) $K_{1} \square K_{2}=K_{1} \triangle K_{2}$ if $K_{2}$ is a two-dimensional square. 
Denote by $B A\left(K_{1} \times K_{2}\right)$ (or simply by $B A$ ) the space of all continuous real-valued biaffine functions on $K_{1} \times K_{2}$, i.e., those which are continuous and affine in each variable. The space $B A$ is, in a natural way, a partially ordered linear space with order unit, and the corresponding state space in its order dual is denoted by $K_{1} \otimes K_{2}$. (This is the projective tensor product of $K_{1}$ and $K_{2}$ defined by Semadeni [11].)

If $f_{1} \otimes f_{2} \in A\left(K_{1}\right) \otimes A\left(K_{2}\right)=A_{1} \otimes A_{2}$, then we can regard $f_{1} \otimes f_{2}$ as an element of $B A\left(K_{1} \times K_{2}\right)$ by

$$
\left(f_{1} \otimes f_{2}\right)\left(x_{1}, x_{2}\right)=f_{1}\left(x_{1}\right) f_{2}\left(x_{2}\right) \text {. }
$$

This embedding of the generating elements of $A_{1} \otimes A_{2}$ extends to a linear embedding of $A_{1} \otimes A_{2}$ into $B A\left(K_{1} \times K_{2}\right)$; we will henceforth regard $A_{1} \otimes A_{2}$ as a subspace of $B A$. Note that this subspace contains the constant functions and separates points of $K_{1} \times K_{2}$. The partial ordering on $A_{1} \otimes A_{2}$ defined by the cone $P^{\wedge}$ is easily seen to be the same as that induced by $B A$, i.e., $\sum_{i=1}^{n} f_{1}^{i} \otimes f_{2}^{i} \in P^{\wedge}$ if and only if $\sum f_{1}^{i}\left(x_{1}\right) f_{2}^{i}\left(x_{2}\right) \geqq 0$ for all $\left(x_{1}, x_{2}\right) \in K_{1} \times K_{2}$. Note, too, that the order duals of each of these spaces coincide with their duals as normed linear spaces, using the supremum norm. Let $\rho: B A\left(K_{1} \times K_{2}\right)^{*} \rightarrow$ $\left[A\left(K_{1}\right) \otimes A\left(K_{2}\right)\right]^{*}$ denote the restriction mapping. If $s \in K_{1} \otimes K_{2}$, then, $\rho(s) \in K_{1} \triangle K_{2}$. It is readily verified (using the extension theorem for positive functionals, cf. [7, p. 8] that $\rho$ is an affine map of $K_{1} \otimes K_{2}$ onto $K_{1} \triangle K_{2}$ and is continuous in the weak * topologies. Let $\omega: K_{1} \times K_{2} \rightarrow$ $K_{1} \otimes K_{2}$ denote the evaluation mapping: If $f \in B A$, then $<\omega\left(x_{1}, x_{2}\right)$, $f>=f\left(x_{1}, x_{2}\right)$ for all $\left(x_{1}, x_{2}\right) \in K_{1} \times K_{2}$. Clearly, $\rho \omega\left(x_{1}, x_{2}\right)=x_{1} \otimes x_{2} \in$ $K_{1} \triangle K_{2}$. The two tensor products $K_{1} \otimes K_{2}$ and $K_{1} \triangle K_{2}$ will be isomorphic if $\rho$ is a bijection; the conditions under which this can occur are considered below. We first investigate further the extreme points of all three tensor products. This has been done before [1, 3, 4], but except for [3] it was assumed that both sets were simplexes.

THEOREM 2.3. Every extreme point of $K_{1} \triangle K_{2}$ [or of $K_{1} \otimes K_{2}$ ] is of the form $x_{1} \otimes x_{2}$ [of the form $\omega\left(x_{1}, x_{2}\right)$ ], where $x_{i} \in \partial K_{i}, i=1,2$. If $\left(x_{1}, x_{2}\right) \in \partial K_{1} \times \partial K_{2}$, then $x_{1} \otimes x_{2}$ is extreme in $K_{1} \square K_{2}$ and in $K_{1} \triangle K_{2}$, and $\omega\left(x_{1}, x_{2}\right)$ is extreme in $K_{1} \otimes K_{2}$.

Proof. Suppose that $M$ is a subspace of $C\left(K_{1} \times K_{2}\right)$ (the continuous real valued functions on $K_{1} \times K_{2}$ ) which contains the constants, so that $M$ is (in the natural ordering) a partially ordered space with order unit. A standard argument shows that every extreme state on $M$ is the restriction of an extreme state on $C\left(K_{1} \times K_{2}\right)$, hence is evaluation at a point $\left(x_{1}, x_{2}\right)$ in $K_{1} \times K_{2}$. If the functions in $M$ are biaffine and separate points, then $x_{i} \in \partial K_{i}, i=1,2$. By applying this 
observation to $M=A_{1} \otimes A_{2}$ and to $M=B A$, we obtain the first part of the theorem.

If $\left(x_{1}, x_{2}\right) \in \partial K_{1} \times \partial K_{2}$, then $x_{1} \otimes x_{2}$ is extreme in $K_{1} \square K_{2}$ by Theorem 2.1. Since $x_{1} \otimes x_{2} \in K_{1} \triangle K_{2} \subset K_{1} \square K_{2}$, we also have $x_{1} \otimes x_{2}$ extreme in $K_{1} \triangle K_{2}$. Since the map $\rho: K_{1} \otimes K_{2} \rightarrow K_{1} \triangle K_{2}$ is a weak* continuous affine surjection, $\rho^{-1}\left(x_{1} \otimes x_{2}\right)$ contains an extreme point of $K_{1} \otimes K_{2}$. By the first part of the theorem, this functional is evaluation at some point of $K_{1} \times K_{2}$ which, since $A_{1} \otimes A_{2}$ separates points of $K_{1} \times K_{2}$, is precisely the point $\left(x_{1}, x_{2}\right)$.

Definition. A Banach space $E$ is said to have the approximation property [2] if for each compact convex subset $C$ of $E$ and each $\varepsilon>0$, there exists a continuous linear transformation (or equivalently, affine transformation) $T: E \rightarrow E$ such that $T(E)$ is finite dimensional and $\|T x-x\|<\varepsilon$ if $x \in C$. It remains open whether every Banach space has the approximation property.

THEOREM 2.4. The restriction mapping $\rho: K_{1} \otimes K_{2} \rightarrow K_{1} \triangle K_{2}$ is one-to-one for every pair of compact convex sets $K_{1}$ and $K_{2}$ if and only if every Banach space has the approximation property.

The proof of this proceeds by a series of simple lemmas. Note that the restriction mapping $\rho$ is a linear operator from $B A\left(K_{1} \otimes K_{2}\right)^{*}$ onto $\left(A_{1} \otimes A_{2}\right)^{*}$ which is the adjoint of the embedding of $A_{1} \otimes A_{2}$ into $B A\left(K_{1} \times K_{2}\right)$. Consequently, $\rho$ will be one-to-one on $B A\left(K_{1} \times K_{2}\right)^{*}$ if and only if $A_{1} \otimes A_{2}$ is dense (in the supremum norm topology) in $B A\left(K_{1} \times K_{2}\right)$. Since $B A\left(K_{1} \times K_{2}\right)^{*}$ is generated by $K_{1} \otimes K_{2}, \rho$ is oneto-one on $B A^{*}$ if and only if it is one-to-one on $K_{1} \otimes K_{2}$.

LEMMA 2.5. Let $K_{1}$ be a compact convex set. If the Banach space $A\left(K_{1}\right)$ has the approximation property, then $A\left(K_{1}\right) \otimes A\left(K_{2}\right)$ is dense in $B A\left(K_{1} \times K_{2}\right)$, for every compact convex set $K_{2}$.

Proof. Suppose that $K_{1}$ and $K_{2}$ are compact convex sets and let $A\left[K_{2}, A\left(K_{1}\right)\right]$ denote the space of all affine continuous mappings $F: K_{2} \rightarrow A\left(K_{1}\right)$ with norm $\|F\|=\sup \left\{\left\|F\left(x_{2}\right)\right\|: x_{2} \in K_{2}\right\}$. If $f \in B A\left(K_{1} \times K_{2}\right)$, then $\left(F x_{2}\right)\left(x_{1}\right)=f\left(x_{1}, x_{2}\right)\left(x_{i} \in K_{i}\right)$ defines an element of $A\left[K_{2}, A\left(K_{1}\right)\right]$, and it is readily verified that the correspondence $f \rightarrow F$ is a linear isometry between these two spaces. Furthermore, under this isometry the subspace $A\left(K_{1}\right) \otimes A\left(K_{2}\right)$ corresponds to the set of those elements in $A\left[K_{2}, A\left(K_{1}\right)\right]$ having finite dimensional range. Suppose, now, that $A\left(K_{1}\right)$ has the approximation property. Given $f$ in $B A\left(K_{1} \times K_{2}\right)$, with corresponding function $F$, let $C=F\left(K_{2}\right)$. This is a compact convex subset of $A\left(K_{1}\right)$, so for any $\varepsilon>0$ there exists a continuous linear 
operator $T: A\left(K_{1}\right) \rightarrow A\left(K_{1}\right)$ such that the range of $T$ is finite dimensional and $\|T g-g\|<\varepsilon$ for each $g$ in $C$. It follows that $T \circ F$ is in $A\left[K_{2}, A\left(K_{1}\right)\right]$, has finite dimensional range, and satisfies $\|T \circ F-F\|<\varepsilon$, so $A_{1} \otimes A_{2}$ is dense in $B A$.

CoRollary 2.6. If $K_{1}$ is a simplex and $K_{2}$ is any compact convex set, then $K_{1} \otimes K_{2}$ is affinely homeomorphic to $K_{1} \triangle K_{2}$.

Proof. It is known [10] that if $K_{1}$ is a simplex, then $A\left(K_{1}\right)^{*}$ is an abstract $(L)$-space. From a theorem of Grothendieck [2], it follows that $A\left(K_{1}\right)$ has the approximation property, so the above lemma and preceding remarks show that $\rho$ is a homeomorphism.

This corollary is also an immediate consequence of a result of Lazar [5, Lemma $3.1 ; 6]$.

Lemma 2.7. If $A\left(K_{1}\right) \otimes A\left(K_{2}\right)$ is dense in $B A\left(K_{1} \times K_{2}\right)$ for each compact convex $K_{2}$, then for each compact convex $C \subset A\left(K_{1}\right)$ and each $\varepsilon>0$, there exists a finite dimensional subspace $M \subset A\left(K_{1}\right)$ and an affine map $\varphi: C \rightarrow M$ such that $\|\varphi(c)-c\|<\varepsilon$ for each $c$ in $C$.

Proof. Let $g \in B A\left(K_{1} \times C\right)$ be defined by $g\left(x_{1}, c\right)=c\left(x_{1}\right)$. By hypothesis there exists $f$ in $A\left(K_{1}\right) \otimes A(C)$ such that $\|f-g\|<\varepsilon$. If $\varphi$ and $\psi$ denote the elements of $A\left[C, A\left(K_{1}\right)\right]$ corresponding to $f$ and $g$ respectively, then $\varnothing$ has range contained in a finite-dimensional subspace $M$ of $A\left(K_{1}\right)$ and $\psi$ is the inclusion map $C \rightarrow A\left(K_{1}\right)$. Hence for each $c$ in $C,\|\varphi(c)-c\|=\|\varphi(c)-\psi(c)\|<\varepsilon$.

Lemma 2.8. Suppose that $E$ is a normed linear space and that $C$ is a nonempty compact convex subset of $E$. Suppose that $\varepsilon>0$ and that there exists a finite dimensional subspace $M \subset E$ and a continuous affine map $\phi: C \rightarrow M$ such that $\|x-\varphi x\|<\varepsilon$ for each $x \in C$. Then there exists a continuous affine map $\psi: E \rightarrow M$ such that $\|x-\psi x\|<2 \varepsilon$ for each $x$ in $C$.

Proof. Let $x_{1}, \cdots, x_{n}$ be a basis for $M$; then there are continuous real valued affine functions $\varphi_{1}, \varphi_{2}, \cdots, \varphi_{n}$ on $C$ such that $\varphi(x)=$ $\sum \varphi_{i}(x) x_{i}$ for $x \in C$. By [9, p. 31] we can choose continuous affine functionals $\psi_{1}, \cdots, \psi_{n}$ on $E$ such that $\left|\varphi_{i}(x)-\psi_{i}(x)\right|<\varepsilon\left(\sum\left\|x_{i}\right\|\right)^{-1}$ for $x$ in $C$. It follows that if $\psi(x)=\sum \psi_{i}(x) x_{i}$ for $x$ in $E$, then $\|\varphi x-\psi x\|<\varepsilon$ for $x \in C$, hence $\|\psi x-x\|<2 \varepsilon$ if $x \in C$.

This result, together with Lemma 2.7, shows that under the density hypothesis of Lemma 2.7, $A\left(K_{1}\right)$ has the approximation 
property.

Lemma 2.9. Suppose that $A(K)$ has the approximation property, for each compact convex set $K$. Then every Banach space $B$ has the approximation property.

Proof. Let $K$ denote the unit ball of $B^{*}$, in its weak* topology. It is easily verified that if $B \times R$ is normed by $\|(x, r)\|=\|x\|+|r|$, then the correspondence between $(x, r)$ and the functional on $K$ defined by $k \rightarrow\langle x, k\rangle+r$ is an isometric isomorphism between $B \times R$ and $A(K)$. Thus, if $C$ is a compact convex subset of $B \subset B \times R$ and $\varepsilon>0$, then there exist a finite dimensional subspace $M$ of $B \times R$ and a continuous linear map $T: B \times R \rightarrow M$ with $\|T x-x\|<\varepsilon$ for $x \in C$. If $P$ denotes the natural projection $P(x, r)=x$ of $B \times R$ onto $B$, then $\|P\|=1$ and the range of $P \circ T$ is finite dimensional in $B$. Furthermore, if $x \in C$ then $\|P(T x)-x\|=\|P(T x-x)\| \leqq\|T x-x\|<\varepsilon$.

This completes the proof of Theorem 2.4.

It has been shown by Lazar [4] and by Davies and Vincent-Smith [1] that if $K_{1}$ and $K_{2}$ are simplexes, then $K_{1} \otimes K_{2}$ is a simplex. (It follows from Corollary 2.6 and Theorem 2.2, of course, that in this case all three tensor products are the same.) The next result shows that the converse is valid. We first require a definition.

A subset $F$ of a compact convex set $K$ is called a face if $F$ is compact convex and if $x, y \in F$ whenever $x, y \in K$ and $\alpha x+(1-\alpha) y \in F$ for some $0<\alpha<1$. It is readily seen that the cone in $A(K)^{*}$ generated by $F$ is a "hereditary" subcone of the cone generated by $K$, hence (cf. [9, p. 64]) if $K$ is a simplex, then so is the face $F$.

Proposition 2.10. If $K_{1}$ and $K_{2}$ are compact convex sets and if any of the sets $K_{1} \otimes K_{2}, K_{1} \square K_{2}$ or $K_{1} \triangle K_{2}$ is a simplex, then $K_{1}$ and $K_{2}$ are simplexes (and the three tensor products are the same).

Proof. Since a face of a simplex is a simplex, it suffices to show that $K_{1}$ is affinely homeomorphic to a face of each of the tensor products. Choose $x_{2}$ in $\partial K_{2}$ and let $F=\left\{x \otimes x_{2} \in K_{1} \square K_{2}: x \in K_{1}\right\}$. It is clear that $F$ is affinely homeomorphic to $K_{1}$; we will show that it is a face of $K_{1} \square K_{2}$. Suppose, then, that $x \otimes x_{2} \in F$ and that $x \otimes x_{2}=\alpha s+(1-\alpha) t$, where $0<\alpha<1$ and $s, t \in K_{1} \square K_{2}$. We have $x=\alpha s_{1}+(1-\alpha) t_{1}$ and $x_{2}=\alpha s_{2}+(1-\alpha) t_{2}$. Since $x_{2}$ is extreme, $s_{2}=$ $x_{2}=t_{2}$. By Lemma 1.1, this implies that $s=s_{1} \otimes x_{2}$ and $t=t_{1} \otimes x_{2}$, which shows that $F$ is a face of $K_{1} \square K_{2}$. It is clear that $F \subset K_{1} \triangle$ $K_{2} \subset K_{1} \square K_{2}$, so $F$ is also a face of $K_{1} \triangle K_{2}$. Finally, the inverse image of $F$ under the restriction map $\rho: K_{1} \otimes K_{2} \rightarrow K_{1} \triangle K_{2}$ is a face 
of $K_{1} \otimes K_{2}$ which is easily seen to be affinely homeomorphic to $K_{1}$; it equals $\left\{\omega\left(x, x_{2}\right): x \in K_{1}\right\}$. The last assertion of the proposition was noted abave. (By "the same," we mean that $K_{1} \triangle K_{2}=K_{1} \square K_{2}$ and that the map $\rho$ is a homeomorphism between $K_{1} \otimes K_{2}$ and $K_{1} \triangle K_{2}$.)

3. Problems and remarks. There remain a few open questions. Although every point of the form $x_{1} \otimes x_{2}$ (with $\left(x_{1}, x_{2}\right) \in \partial K_{1} \times \partial K_{2}$ ) is extreme in $K_{1} \square K_{2}$, there will be additional extreme points if neither of the sets is a simplex and (for instance) one of them is a square. (These additional points are easily seen to be outside of the set $\left\{x_{1} \otimes x_{2}:\left(x_{1}, x_{2}\right) \in K_{1} \times K_{2}\right\}$.) Is there a simple description of these points?

Another question is related to the characterization of simplexes in terms of affine cross-sections for the resultant map. Suppose that such a cross-section $\sigma$ exists for the compact convex set $K$ (so that $K$ is a simplex); must $\sigma$ coincide with the inverse of $r_{m}$ ? Equivalently, does $\sigma$ map $K$ into the maximal measures $Q(K)$ ? [Since $\sigma(x)$ is necessarily the maximal measure $\varepsilon_{x}$ whenever $x \in \partial K$, we have $\sigma(\operatorname{conv} \partial K) \subset$ $Q(K).]^{1}$

It should also be noted that it is possible that $K_{1} \triangle K_{2}$ is always a face of $K_{1} \square K_{2}$. This is suggested by the fact (Theorem 2.3) that $\partial\left(K_{1} \triangle K_{2}\right) \subset \partial\left(K_{1} \square K_{2}\right)$.

We have dealt solely with tensor products of two spaces (or of two convex sets). Those results which can be formulated for finite tensor products can, however, be readily proved by appropriate induction arguments and can then be extended by standard methods to infinite tensor products. The techniques for doing this are well known $[1,3,4]$ so we have restricted ourselves to the simplest case in order to exhibit the essential ideas of the proofs. Similarly, these results can be extended fairly easily to sets of states which are invariant under appropriate actions of semigroups, as was done in [3]. (The extension of Theorem 1.4 to more than two spaces requires that all but one of the state spaces be simplexes.)

We wish to thank Professors A. Lazar and E. Effros for several helpful conversations on the subject matter of this paper.

\section{BIBLIOGRAPHY}

1. E. B. Davies and G. F. Vincent-Smith, Tensor products, infinite products and projective limits of Choquet simplexes, Math. Scand. 22 (1968), 145-164.

2. A. Grothendieck, Produits tensoriels topologiques et espaces nucléaires, Memoirs Amer. Math. Soc. 16 (1955).

1 Added proof: Hicham Fakhoury proved that an affine cross-section, if it exists, is unique. [C. R. Acad. Sci. Paris, 269 Série A (1969), 21-24]. 
3. A. Hulanicki and R. R. Phelps, J. Functional Analysis 2 (1968), 177-201.

4. A. Lazar, Affine products of simplexes, Math. Scand. 22 (1968), 165-175.

5. - Spaces of affine continuous functions on simplexes, Trans. Amer. Math. Soc. 134 (1968), 503-525.

6. Affine functions on simplexes and extreme operators, Israel J. Math. 5 (1967), 31-43.

7. Isaac Namioka, Partially ordered linear topological spaces, Memoirs Amer. Math. Soc. 24 (1957).

8. A. L. Peressini and D. R. Sherbert, Ordered topological tensor products, Proe. Lond. Math. Soc. (1968).

9. R. R. Phelps, Lectures on Choquet's theorem, D. Van Nostrand, Princeton, N. J., 1966.

10. Z. Semadeni, Free compact convex sets, Bull. Acad. Polon. Sci. Ser. Sci., Math., Astr. et Phys. 13 (1964), 141-146.

11. Categorical methods in convexity, Proc. Colloq. on Convexity, Copenhagen, 1965, 281-307.

Received October 4, 1968. Research on this paper supported in part by NSF Grants GP 8348 and GP 7164, respectively.

UNIVERSITY OF WASHINGTON 


\section{PACIFIC JOURNAL OF MATHEMATICS}

\section{EDITORS}

\author{
H. ROYDEN \\ Stanford University \\ Stanford, California \\ Richard Pierce \\ University of Washington \\ Seattle, Washington 98105
}

\author{
J. DugundJI \\ Department of Mathematics \\ University of Southern California \\ Los Angeles, California 90007 \\ BASIL GoRDON \\ University of California \\ Los Angeles, California 90024
}

\section{ASSOCIATE EDITORS}
E. F. BECKENBACH
B. H. Neumann
F. WOLF
K. YoSHIDA

\section{SUPPORTING INSTITUTIONS}

\author{
UNIVERSITY OF BRITISH COLUMBIA \\ CALIFORNIA INSTITUTE OF TECHNOLOGY \\ UNIVERSITY OF CALIFORNIA \\ MONTANA STATE UNIVERSITY \\ UNIVERSITY OF NEVADA \\ NEW MEXICO STATE UNIVERSITY \\ OREGON STATE UNIVERSITY \\ UNIVERSITY OF OREGON \\ OSAKA UNIVERSITY \\ UNIVERSITY OF SOUTHERN CALIFORNIA
}

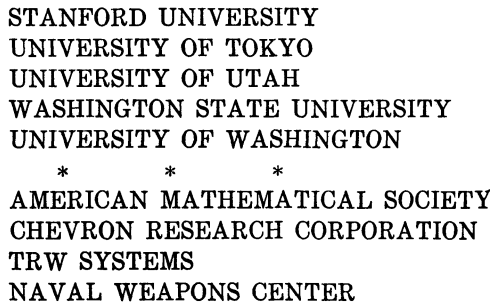

The Supporting Institutions listed above contribute to the cost of publication of this Journal, but they are not owners or publishers and have no responsibility for its content or policies.

Mathematical papers intended for publication in the Pacific Journal of Mathematics should be in typed form or offset-reproduced, double spaced with large margins. Underline Greek letters in red, German in green, and script in blue. The first paragraph or two must be capable of being used separately as a synopsis of the entire paper. It should not contain references to the bibliography. Manuscripts, in duplicate if possible, may be sent to any one of the four editors. Please classify according to the scheme of Math. Rev. 36, 1539-1546. All other communications to the editors should be addressed to the managing editor, Richard Arens, University of California, Los Angeles, California, 90024.

50 reprints are provided free for each article; additional copies may be obtained at cost in multiples of 50 .

The Pacific Journal of Mathematics is published monthly. Effective with Volume 16 the price per volume (3 numbers) is $\$ 8.00$; single issues, $\$ 3.00$. Special price for current issues to individual faculty members of supporting institutions and to individual members of the American Mathematical Society: $\$ 4.00$ per volume; single issues $\$ 1.50$. Back numbers are available.

Subscriptions, orders for back numbers, and changes of address should be sent to Pacific Journal of Mathematics, 103 Highland Boulevard, Berkeley, California, 94708.

PUBLISHED BY PACIFIC JOURNAL OF MATHEMATICS, A NON-PROFIT CORPORATION

Printed at Kokusai Bunken Insatsusha (International Academic Printing Co., Ltd.), 7-17, Fujimi 2-chome, Chiyoda-ku, Tokyo, Japan. 


\section{Pacific Journal of Mathematics}

\section{Vol. 31, No. $2 \quad$ December, 1969}

Efraim Pacillas Armendariz, Quasi-injective modules and stable torsion

classes..........................................

J. Adrian (John) Bondy, On Ulam's conjecture for separable graphs...

Vasily Cateforis and Francis Louis Sandomierski, On commutative rings over which the singular submodule is a direct summand for every module .....

Rafael Van Severen Chacon, Approximation of transformations with continuous

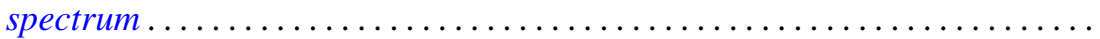

Raymond Frank Dickman and Alan Zame, Functionally compact spaces ...... 303

Ronald George Douglas and Walter Rudin, Approximation by inner

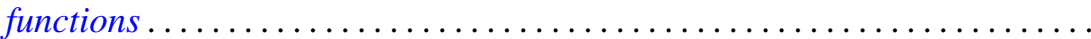

John Walter Duke, A note on the similarity of matrix and its conjugate

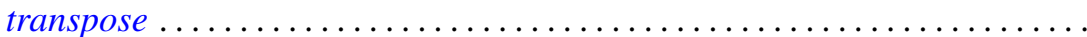

Micheal Neal Dyer and Allan John Sieradski, Coverings of mapping

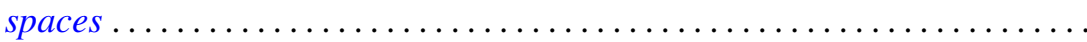

Donald Campbell Dykes, Weakly hypercentral subgroups of finite groups .....

Nancy Dykes, Mappings and realcompact spaces.....................

Edmund H. Feller and Richard Laham Gantos, Completely injective

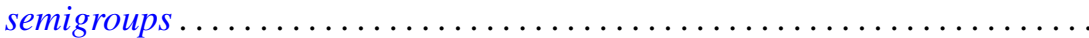

Irving Leonard Glicksberg, Semi-square-summable Fourier-Stieltjes

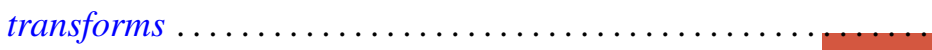

Samuel Irving Goldberg and Kentaro Yano, Integrability of almost cosymplectic structures...

Seymour Haber and Charles Freeman Osgood, On the sum $\sum\langle n \alpha\rangle^{-t}$ and numerical integration ..........................

Sav Roman Harasymiv, Dilations of rapidly decreasing functions ....

William Leonard Harkness and R. Shantaram, Convergence of a sequence of

transformations of distribution functions

Herbert Frederick Kreimer, Jr., A note on the outer Galois theory of rings ...

James Donald Kuelbs, Abstract Wiener spaces and applications to analysis. .

Roland Edwin Larson, Minimal $T_{0}$-spaces and minimal $T_{D}$-spaces...

A. Meir and Ambikeshwar Sharma, On Ilyeff's conjecture .

Isaac Namioka and Robert Ralph Phelps, Tensor products of compact convex sets....

James L. Rovnyak, On the theory of unbounded Toeplitz operators ....

Benjamin L. Schwartz, Infinite self-interchange graphs.......

George Szeto, On the Brauer splitting theorem...

Takayuki Tamura, Semigroups satisfying identity $x y=f(x$,

Kenneth Tolo, Factorizable semigroups .................. 